\title{
Phosphorylation of the $\beta^{\prime}$ Subunit of RNA Polymerase and Other Host Proteins upon $\phi \mathrm{Cd} 1$ Infection of Caulobacter crescentus
}

\author{
DAVID HODGSON,$\dagger$ LUCILLE SHAPIRO, AND KEI AMEMIYA* \\ Department of Molecular Biology, Division of Biological Sciences, Albert Einstein College of Medicine, Bronx, \\ New York 10461
}

Received 25 February 1985/Accepted 7 March 1985

\begin{abstract}
A protein kinase activity is induced early after infection of Caulobacter crescentus by the DNA phage $\phi$ Cd1. After phage infection at least 40 proteins are phosphorylated; these include DNA-binding proteins, a membrane-associated protein, and several ribosomal proteins. One of the phosphorylated DNA-binding proteins was identified as the $\beta^{\prime}$ subunit of the host RNA polymerase.
\end{abstract}

$\phi \mathrm{Cd} 1$ is a small lytic phage which infects the dimorphic bacterium Caulobacter crescentus (16). Its genome is organized in two functional regions containing early and late genes, respectively $(2,3)$. The host RNA polymerase is required for the expression of genes in the early region, and a rifampin-resistant RNA polymerase, which appears to be an early phage-gene product, is responsible for the expression of genes in the late region $(2,13)$. De novo RNA and protein synthesis is essential for the appearance of the rifampin-resistant RNA polymerase activity. The switch from early to late gene transcription in $C$. crescentus phage $\phi C d 1$ represents a clearly defined system in which to study the regulation of differential gene expression. Proteins other than RNA polymerases have been implicated in the regulation of this switch. A coliphage T7-encoded protein kinase, itself an early gene product, has been shown to be required in vivo for correct termination signal recognition by the host RNA polymerase (12). Because it has been suggested that a protein kinase activity is present in $\phi C d 1$-infected $C$. crescentus cells (16), we examined the phosphorylated proteins which appear after $\phi \mathrm{Cd} 1$ infection.

Approximately 40 phosphorylated proteins could be detected in cell extracts $10 \mathrm{~min}$ after phage infection of $C$. crescentus (Fig. 1). Additional protein phosphorylation was observed if infection was allowed to continue for $30 \mathrm{~min}$. Parallel samples treated with protease showed a decrease in the size of the radioactive bands, indicating that proteins were being phosphorylated (data not shown). Except for two phosphorylated proteins of apparent molecular weights of 94,000 and 50,000 ( $94 \mathrm{~K}$ and $50 \mathrm{~K}$ proteins), which were shown to be present in extracts from uninfected cells (see Fig. 3A), protein phosphorylation was inhibited if chloramphenicol $(100 \mu \mathrm{g} / \mathrm{ml})$ was added to the culture just before phage infection. (Fig. 1, lane 7). Furthermore, the addition of rifampin to the cells before the addition of phage completely inhibited the appearance of phage-specific phosphorylated proteins, whereas the addition of rifampin to the cells shortly after phage infection did not inhibit protein phosphorylation. These results suggest that de novo RNA and protein synthe-

\footnotetext{
* Corresponding author.

$\dagger$ Present address: Department of Biochemistry, Stanford University School of Medicine, Stanford, CA 94305.
}

sis is required for protein phosphorylation after phage infection. A protein kinase activity which may be responsible for the phosphorylation of the $94 \mathrm{~K}$ and $50 \mathrm{~K}$ proteins was previously identified in uninfected $C$. crescentus cells (1). The phosphorylation of a large group of proteins after phage infection suggests that $\phi \mathrm{Cd} 1$ induces or encodes an additional protein kinase.

To identify the proteins phosphorylated after $\phi \mathrm{Cd} 1$ infection, we examined various protein fractions from extracts of ${ }^{32} \mathrm{P}$-labeled, phage-infected cells. Host RNA polymerase was immunoprecipitated with anti-RNA polymerase antiserum and analyzed by sodium dodecyl sulfate-polyacrylamide gel electrophoresis (SDS-PAGE) (Fig. 2A). Autoradiograms of the separated RNA polymerase subunits showed that the $\beta^{\prime}$ subunit was specifically phosphorylated (Fig. 2A, lane 2). To determine if other DNA-binding proteins were phosphorylated, extracts from infected cells were passed through a heparin-agarose column. The peak protein fractions eluted from this column were combined and loaded onto a denatured DNA-cellulose column. The protein profile of a portion of the peak protein fraction eluted from the DNAcellulose column is shown in Fig. 2B. Approximately eight proteins were phosphorylated, ranging in apparent molecular weight from 165,000 to 27,000 with the $115 \mathrm{~K}, 64 \mathrm{~K}$, and $20 \mathrm{~K}$ proteins being the most heavily phosphorylated. The phosphorylated $165 \mathrm{~K}$ protein comigrated with the $\beta^{\prime}$ subunit of RNA polymerase that had been previously identified by immunoprecipitation with anti-RNA polymerase antisera. The phosphoproteins eluted from the DNA-cellulose column were not detected in parallel experiments with uninfected cell extracts (data not shown).

Examination of membrane and ribosomal protein fractions from infected cells by SDS-PAGE showed the presence of phosphorylated proteins (data not shown). Both the outer and inner membrane fractions (14) contained an 81,000- and a 41,000-molecular-weight phosphorylated protein. However, the phosphorylated $41 \mathrm{~K}$ protein was present only in $\phi C d 1$-infected cells, whereas the $81 \mathrm{~K}$ phosphoprotein was present in both infected and uninfected cell extracts. Ribosomes isolated (15) from infected cells contained at least seven phosphoproteins $(94 \mathrm{~K}, 84 \mathrm{~K}, 68 \mathrm{~K}, 37 \mathrm{~K}, 29 \mathrm{~K}, 19 \mathrm{~K}$, and $17 \mathrm{~K}$ proteins). Two of these phosphorylated ribosomal proteins $(68 \mathrm{~K}$ and $29 \mathrm{~K})$ were phosphorylated in the absence of 


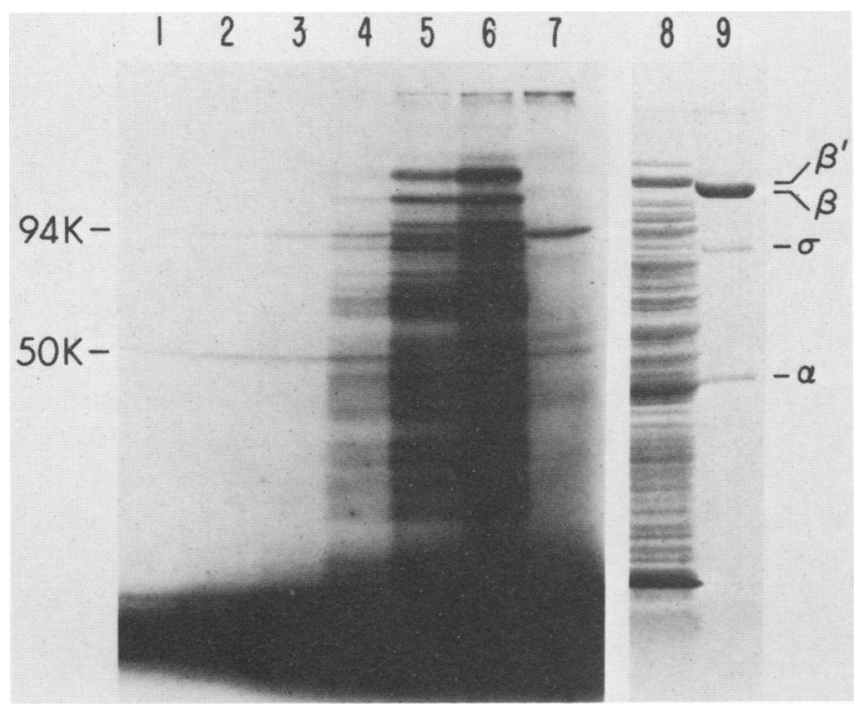

FIG. 1. SDS-PAGE of ${ }^{32}$ P-labeled phosphoproteins extracted from $\phi C \mathrm{~d} 1$-infected $C$. crescentus cells. $C$. crescentus was infected with $\phi \mathrm{Cd} 1$ at a multiplicity of infection of 20 to 50 in the presence of ${ }^{32} \mathrm{P},(200 \mu \mathrm{Ci} / \mathrm{ml})$ for $20 \mathrm{~min}$ in one-fourth-diluted peptone-yeast extract (11). Samples $(5 \mathrm{ml})$ were removed at the indicated times after infection, and extracts were prepared, treated with RNase I and DNase I, and analyzed by SDS-PAGE (10\%). Lanes 1 through 6 are autoradiograms of phosphoproteins extracted $1,3,5,10,15$, and $30 \mathrm{~min}$ after phage infection, respectively. Lanes 7 and 8 show an autoradiogram and a Coomassie blue stain, respectively, of proteins from cultures which were incubated with chloramphenicol $(50 \mu \mathrm{g} / \mathrm{ml})$ for $5 \mathrm{~min}$ before addition of ${ }^{32} \mathrm{P}$, and $\phi \mathrm{Cd} 1$ and then incubated for an additional $30 \mathrm{~min}$. Lane 9 shows purified $C$. crescentus RNA polymerase stained with Coomassie blue. The Coomassie blue-stained protein pattern of lanes 1 through 6 appeared the same as that of lane 8. Phosphoprotein molecular weights were determined by comparison with the migration of phosphorylase $b(94,000)$, albumin $(67,000)$, ovalbumin $(43,000)$, carbonic anhydrase $(30,000)$, trypsin inhibitor $(20,000), \alpha$-lactalbumin $(14,400)$, and subunits of RNA polymerase (5) (shown on the right).

$\phi \mathrm{Cd} 1$ infection. Of the proteins which were specifically phosphorylated after phage infection, the $37 \mathrm{~K}$ and $17 \mathrm{~K}$ proteins were the most heavily phosphorylated.

The nature of the phosphate bonds on the $94 \mathrm{~K}$ and $50 \mathrm{~K}$ proteins and on the proteins phosphorylated after phage infection was investigated by determining their sensitivity to alkaline phosphatase. A phosphate ester bond is sensitive to cleavage by alkaline phosphatase but an acyl phosphate bond is not (1). Accordingly, extracts of uninfected cells (Fig. 3A) and $\phi C d 1$-infected cells (Fig. 3B) grown in the presence of ${ }^{32} \mathrm{P}$, were treated with alkaline phosphatase. Of the two major phosphoproteins observed in uninfected cell extracts (94K and $50 \mathrm{~K}$ ), the $50 \mathrm{~K}$ phosphoprotein was insensitive to alkaline phosphatase and the $94 \mathrm{~K}$ protein was only partially sensitive (Fig. 3A), suggesting that a portion of the phosphate residues on these proteins is attached either by an acyl phosphate bond or by protected ester bonds. Two minor phosphoproteins ( $90 \mathrm{~K}$ and $67 \mathrm{~K}$ ) were susceptible to alkáline phosphatase treatment, and thus their phosphoprotein bonds may differ from those on the $50 \mathrm{~K}$ phosphoprotein. All but two of the phosphoproteins which were specifically observed in phage-infected cells were cleaved by alkaline phosphatase (Fig. 3B). The two alkaline phosphatase-resistant proteins $(44 \mathrm{~K}$ and $41 \mathrm{~K})$, which were not present in uninfected extracts (Fig. 3A), may be phage- induced proteins which are phosphorylated by the endogenous $C$. crescentus phosphoacyl kinase (1).

The nature of the phosphate bond on the $\beta^{\prime}$ subunit of RNA polymerase was determined after purification of the enzyme from ${ }^{32} \mathrm{P}$-labeled phage-infected cells by DNA-cellulose chromatography (5). The purified enzyme fraction, which contained RNA polymerase core enzyme and a 64,000-molecular-weight DNA-binding protein (Fig. 3C), was treated with alkaline phosphatase before SDS-PAGE (Fig. 3C, lane 3). The phosphate groups on the $\beta^{\prime}$ subunit of RNA polymerase and on the $64 \mathrm{~K}$ protein were cleaved by alkaline phosphatase, suggesting that these phosphoproteins are phosphate esters. The phosphorylated amino acid(s) on the RNA polymerase $\beta^{\prime}$ subunit was identified after isolation of the RNA polymerase by immunoprecipitation with antiRNA polymerase antibody and gel electrophoresis. The $\boldsymbol{\beta}^{\prime}$ subunit was eluted from the gel, amino acids were prepared essentially as described previously $(8,9)$, and phosphoamino acids were identified by paper electrophoresis (Fig. 4). The phosphorylated amino acid from the $\beta^{\prime}$ subunit comigrated primarily with a phosphothreonine standard. Some fraction may have migrated with phosphoserine. It appears, therefore, that the protein kinase activity induced upon $\phi \mathrm{Cd} 1$ infection catalyzes the formation of a phosphate ester linkage primarily to a threonine residue.

Phosphorylation of the $\beta^{\prime}$ subunit of the $C$. crescentus RNA polymerase by the phage $\phi C d 1$-induced kinase suggests that one function of this kinase activity may be to affect the host transcription processes. In addition, phosphorylation of ribosomal and other host proteins after $\phi \mathrm{Cd} 1$ infection of $C$. crescentus suggests that alterations in the host

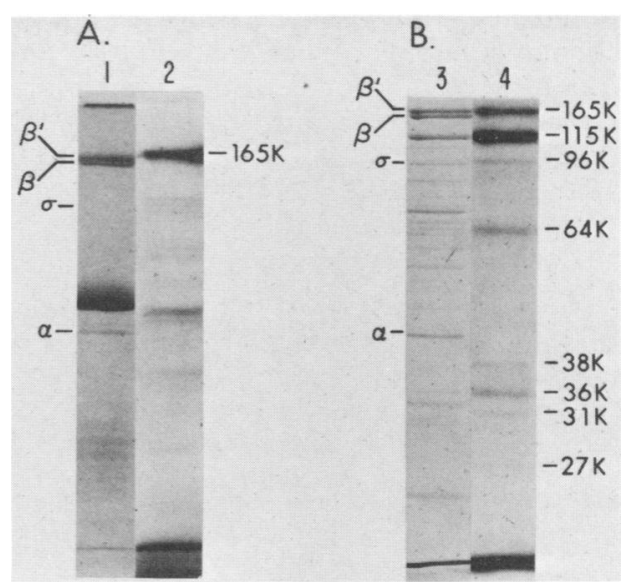

FIG. 2. Phosphorylation of the host RNA polymerase and DNAbinding proteins after infection with $\phi \mathrm{Cd} 1$. $C$. crescentus cells were infected with $\phi \mathrm{Cd} 1$ for $20 \mathrm{~min}$ in the presence of ${ }^{32} \mathrm{P}$, and extracts were prepared as described in the legend to Fig. 1 . The final samples were analyzed by SDS-PAGE (10 to $20 \%$ gradient). (A) Analysis of ${ }^{32} \mathrm{P}$-labeled proteins after immunoprecipitation with anti-RNA polymerase antibody (14). (B) Analysis of ${ }^{32} \mathrm{P}$-labeled proteins which bind to DNA-cellulose. To examine proteins which bind to DNAcellulose, an extract from infected cells was first loaded onto a heparin-agarose column, and proteins were then eluted from the column with a salt gradient (17). Fractions containing the major protein peaks were combined, dialyzed, and loaded onto a DNAcellulose column. Proteins were eluted from the column with a salt gradient (5), and a portion of the fraction containing the peak protein concentration was examined. In panels $A$ and $B$, lanes 1 and 3 represent the Coomassie blue-stained gel and lanes 2 and 4 represent the autoradiogram. 

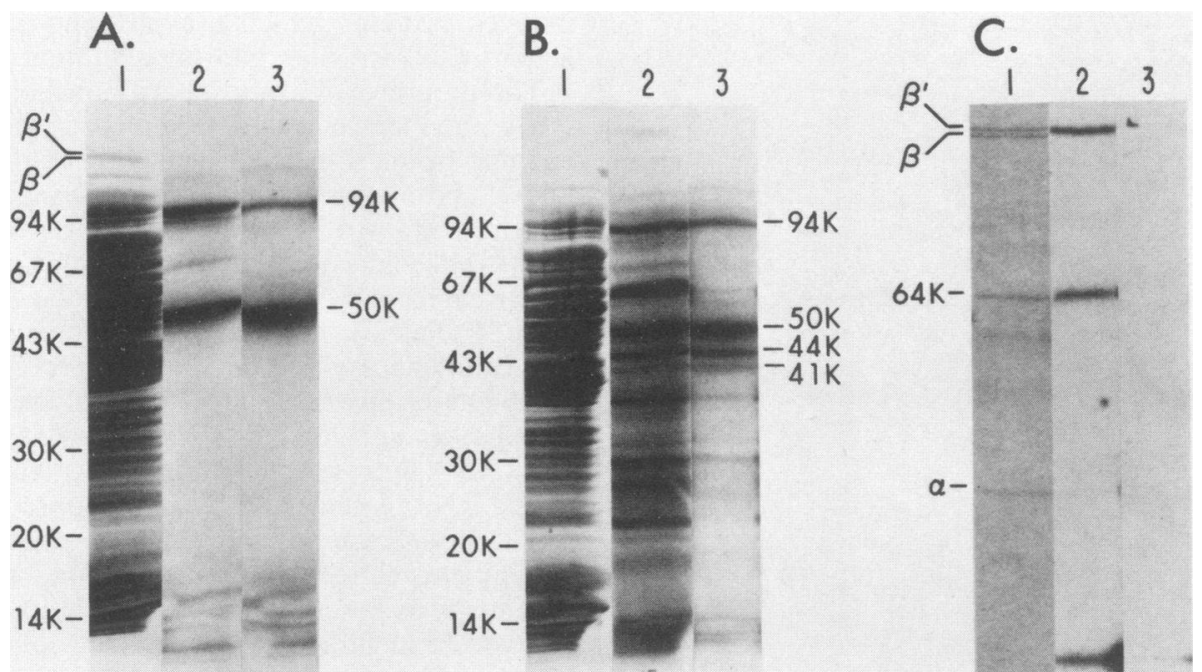

FIG. 3. Effect of alkaline phosphatase on ${ }^{32} \mathrm{P}$-labeled proteins from uninfected and phage-infected cells. ${ }^{32} \mathrm{P}$-labeled proteins $(200 \mu \mathrm{g})$ from uninfected (A) and $\phi C d 1$-infected (B) cells were prepared as described in the legend to Fig. 1, except that nucleic acids were removed by a phase partition procedure (5) before treatment with alkaline phosphatase. Although the phase-partitioning procedure excluded the RNA polymerase subunits from only the infected cell extracts, it was a necessary procedure because nucleic acids are competitive substrates for alkaline phosphatase and the maximum yield of protein was desired. (A) ${ }^{32}$ P-labeled RNA polymerase preparation (0.5 $\left.\mu \mathrm{g}\right)(\mathrm{c})$ was obtained by DNA-cellulose chromatography. Samples were analyzed by SDS-PAGE (10\%). In each panel, lane 1 shows a Coomassie blue stain of the autoradiogram shown in lane 2 , and lane 3 shows an autoradiogram of phosphoproteins treated with calf intestinal alkaline phosphatase $(75,100$ and $75 \mathrm{U}$ for $\mathrm{A}, \mathrm{B}$, and $\mathrm{C}$, respectively) in alkaline phosphatase buffer (10 mM Tris-hydrochloride, [pH 8.0$], 1 \mathrm{mM} \mathrm{MgCl}$ plus $0.1 \mathrm{mM}$ $\mathrm{ZnCl}_{2}$ ) for $60 \mathrm{~min}$ at $37^{\circ} \mathrm{C}$.

may occur at the level of translation or posttranslation. In fact, coliphage $\mathrm{T} 7$ protein kinase has been implicated in the posttranscriptional regulation of $\mathrm{T} 7$ gene expression. RNase III activity was found to be stimulated fourfold when the cells were infected with wild-type phage T7 but not stimu-

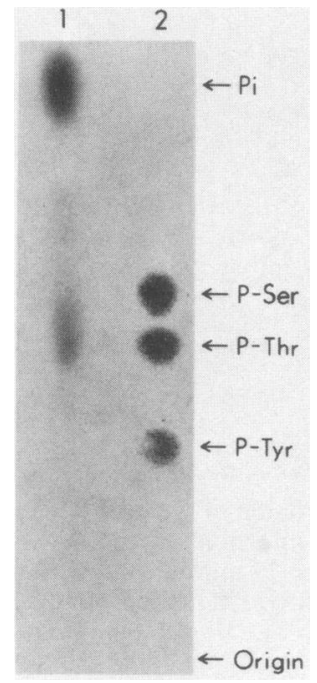

FIG. 4. Identification of the phosphorylated amino acid of the $\beta^{\prime}$ subunit of RNA polymerase isolated from $\phi C d 1$-infected cells. ${ }^{32} \mathrm{P}$-labeled RNA polymerase was purified by immunoprecipitation with anti-RNA polymerase antibody. The $\beta^{\prime}$ subunit was isolated by SDS-PAGE and acid hydrolyzed, and phosphoamino acid was analyzed by paper electrophoresis under conditions described by Enami and Ishihama (8). Lane 1 is an autoradiogram of the phosphorylated amino acid derived from the RNA polymerase $\beta^{\prime}$ subunit. Lane 2 shows standards $(10 \mu \mathrm{mol}$ each) visualized by ninhydrin staining: phosphoserine (P-Ser), phosphothreonine (PThr), and phosphotyrosine (P-Tyr). lated when cells were infected with a $\mathrm{T} 7$ protein kinase mutant (10). RNase III in Escherichia coli processes the T7 early polycistronic mRNA synthesized by the host RNA polymerase (7). Recently, we have shown that the $C$. crescentus RNase III processes the early $\phi \mathrm{Cd} 1 \mathrm{mRNA}$ synthesized in vitro $(4,6)$ and have observed that one of the phosphoproteins associated with the ribosomal fraction from $\phi C d 1$-infected cells is similar in size $(19,000$ molecular weight) to the $C$. crescentus RNase III. However, the function of the $\phi C d 1$-induced protein kinase in $C$. crescentus still remains to be elucidated.

\section{LITERATURE CITED}

1. Agabian, N., O. M. Rosen, and L. Shapirio. 1972. Characterization of a protein acyl kinase from Caulobacter crescentus. Biochem. Biophys. Res. Commun. 49:1690-1698.

2. Amemiya, K., B. Raboy, and L. Shapiro. 1980. Involvement of the host RNA polymerase in the early transcription program of Caulobacter crescentus bacteriophage $\phi C \mathrm{~d} 1$ DNA. Virology 104:109-116.

3. Amemiya, K., and L. Shapiro. 1982. In vitro transcription of the early region of Caulobacter phage $\phi C \mathrm{~d} 1$ deoxyribonucleic acid by host RNA polymerase. Biochemistry 21:4707-4713.

4. Amemiya, K., and L. Shapiro. 1983. Differential template recognition by the Caulobacter crescentus and the Escherichia coli RNA polymerase. J. Biol. Chem. 258:8984-8992.

5. Amemiya, K., C. W. Wu, and L. Shapiro. 1977. Caulobacter crescentus RNA polymerase. Purification of holoenzyme and core polymerase. J. Biol. Chem. 252:4157-4165.

6. Bellofatto, V., K. Amemiya, and L. Shapiro. 1983. Purification and characterization of an RNA processing enzyme from Caulobacter crescentus. J. Biol. Chem. 258:5467-5476.

7. Dunn, J. J., and F. W. Studier. 1973. T7 early RNAs and Escherichia coli ribosomal RNAs are cut from large precursor RNAs in vivo by ribonuclease III. Proc. Natl. Acad. Sci. U.S.A. 70:3296-3300.

8. Enami, M., and A. Ishihama. 1984. Protein phosphorylation in Escherichia coli purification of a protein kinase. J. Biol. Chem. 259:526-533. 
9. Huttner, W. B., and P. Greengard. 1979. Multiple phosphorylation sites in protein I and their differential regulation by cyclic AMP and calcium. Proc. Natl. Acad. Sci. U.S.A. 76:5402-5406.

10. Mayer, J. E., and M. Schweiger. 1983. RNase III is positively regulated by T7 protein kinase. J. Biol. Chem. 258:5340-5343.

11. Poindexter, J. S. 1964. Biological properties and classification of the Caulobacter group. Bacteriol. Rev. 28:231-295.

12. Ponta, H., H. J. Rahmsdorf, S. H. Pai, M. Hirsch-Kauffmann, P. Herrlich, and M. Schweiger. 1974. Control of gene expression in bacteriophage T7: transcriptional controls. Mol. Gen. Genet. 134:281-287.

13. Raboy, B., L. Shapiro, and K. Amemiya. 1980. Physical map of Caulobacter crescentus bacteriophage $\phi \mathrm{Cd} 1 \mathrm{DNA}$. J. Virol. 34:542-549.
14. Shapiro, L., J. Mansour, P. Shaw, and S. Henry. 1982. Synthesis of specific membrane proteins is a function of DNA replication and phospholipid synthesis in Caulobacter crescentus. J. Mol. Biol. 150:303-322.

15. Traub, P., S. Mizushima, C. V. Lowry, and M. Nomura. 1971. Reconstitution of ribosomes from subribosomal components. Methods Enzymol. 20:391-407.

16. West, D., C. Lagenaur, and N. Agabian. 1976. Isolation and characterization of Caulobacter crescentus bacteriophage $\phi C d 1$. J. Virol. 17:568-575.

17. Wiggs, J. L., M. Z. Gilman, and M. J. Chamberlin. 1981. Heterogeneity of RNA polymerase in Bacillus subtilis: evidence for an additional factor in vegetative cells. Proc. Natl. Acad. Sci. U.S.A. 78:2762-2766. 